\title{
EDUCATION
}

\section{La adaptación escolar de los menores adoptados. Riesgos y estrategias de intervención}

\section{Ana Ma Rosser Limiñana y Raquel Suriá Martínez}

Departamento de Comunicación y Psicología Social Universidad de Alicante. Alicante.

\section{España}

Correspondencia: Ana $M^{a}$ Rosser Limiñana. Universidad de Alicante. Campus de S. Vicente, s/n. San Vicente del Raspeig 03690 (Alicante). España. E-mail: ana.rosser@ua.es

(C) Education \& Psychology I+D+i y Editorial EOS (España) 


\section{Resumen}

Introducción. A lo largo de este trabajo se pretenden analizar las peculiaridades del ajuste escolar en los menores adoptados. Estos menores llegan a una adopción tras haber sufrido experiencias más o menos traumáticas, que pueden estar condicionando su desarrollo y sus aprendizajes. Al llegar a su nueva familia, deben adaptarse a un nuevo contexto, el escolar, donde deben relacionarse con otros niños y adultos, y adaptarse a nuevas rutinas.

Método. Los resultados forman parte de un estudio más amplio realizado con 133 menores adoptados en España. Su objetivo ha sido evaluar las dificultades que presentaban los menores a la llegada a su nueva familia, los factores que pueden estar influyendo en su aparición, y su evolución tras varios años de convivencia.

Resultados. Se comprueba que los menores adoptados presentan dificultades en diferentes ámbitos, entre ellos el ajuste escolar, y que existe una relación directa entre las dificultades y sus experiencias previas a la adopción, así como entre estas dificultades y los problemas afectivo-emocionales o de comportamiento. Igualmente se aprecia una recuperación, aunque incompleta, en sus rendimientos en el momento del estudio.

Conclusiones: Se proponen actuaciones desde el servicio post-adoptivo para favorecer la adaptación de los menores a la escuela.

Palabras clave: adopción, niños adoptados, adaptación escolar, post-adopción 


\title{
School adaptation of adopted minors: Risks and intervention strategies
}

\begin{abstract}
Introduction. Along the present paper, we want to analyze some of the peculiarities of the school adaptation in the adopted children. Children arrive to an adoption having suffered more or less traumatic experiences that can condition their development and learning. At their arrival to their new family, the minors have to adapt themselves not only to their new family, but also to a new context, the school, where they have to mix with children and adults, and to adapt themselves to a new routine.

Method. The results are based on a wider research carried out with 133 minors adopted in Spain and which aim has been to evaluate the difficulties presented by the children at their arrival to their new family, the factors that can play a role in triggering them and their evolution after some years living together.

Results. It has been checked that the adopted minors show difficulties in different areas, being included the school, and that it exists a direct connection between the difficulties presented by the minors and their experiences before the adoption and between these difficulties and the behavioral or emotional problems. Likewise, a recovery, even incomplete, in their school performance can be seen.
\end{abstract}

Conclusions. Some actions are proposed from the post-adoptive service to favor the adaptation of the minors to the school.

Keywords: adoption, adopted children, school adaptation, post-adoption 


\section{Introducción}

La adopción es una medida de protección a la infancia que pretende proporcionar una familia a aquellos menores que han sido separados de la propia por desatención, maltrato o abandono y con los que se considera inviable el retorno. La investigación sobre el tema señala que muchos de los niños y niñas que llegan a una adopción han sufrido experiencias negativas, falta de cuidados y oportunidades, y han vivido separaciones y rupturas más o menos traumáticas, periodos de institucionalización o de acogida con otras familias distintas a la adoptiva. Todo ello ha dejado su huella, condicionando su desarrollo, sus aprendizajes, su forma de entender el mundo y de relacionarse con los demás. Es fácil suponer que, como consecuencia de todo ello, estos menores pueden encontrase con dificultades en su proceso de adaptación (Brodzinsky, 1990; O’Connor, Rutter, Beckett, Keaveney, Kreppner, and the ERA study team, 2000; Rutter, 200). En concreto, en la escuela, donde el menor se encuentra con nuevas exigencias, pautas, horarios, nuevos conocimientos, evaluaciones, etc., estas carencias y dificultades se hacen más evidentes. Inmadurez, problemas de atención, reacciones emocionales desproporcionadas, inseguridad o falta de conocimientos básicos, así como dificultades en el idioma cuando vienen de un país extranjero, son fenómenos cotidianos en su tiempo escolar (Verlust, Althaus y Verluis-Den, 1990).

Son muchos los factores previos a la adopción que se ha comprobado que influyen en el desarrollo cognitivo de los niños y niñas que llegan a los sistemas de protección infantil y que repercuten en su adaptación escolar. Así, diversos estudios han documentado que el peso bajo al nacer, la desnutrición prenatal, o la exposición prenatal al alcohol tienen un efecto negativo en su desarrollo cognitivo (Colombo, Parra y López, 1992; Gindis, 1998; Grantham-McGregor, 1995; McGuiness y Pallansch, 2000; Miller, 2000; Mitchel, 2001). También la institucionalización ha demostrado tener consecuencias negativas para el desarrollo de los niños (Beckett et al, 2006; Zeanah et al, Fox, Smyke, Marshall, Parker y Koga, 2003). Es difícil que un entorno institucional pueda ofrecer las experiencias adecuadas necesarias para apoyar el desarrollo óptimo del cerebro (Rutter, 2005), incluso cuando estas instituciones se encuentran en las mejores condiciones. La falta de relaciones consistentes y duraderas con adultos de referencia dificulta así mismo el establecimiento de unas relaciones de apego seguras, afectando su desarrollo emocional y social. (O'Connor et al, 2000; Verhulst, Althaus y Verluis-den Bierman, 1990, 1992; Zeanah et al, 2003). Estos problemas también repercuten 
en las funciones cognitivas y del desarrollo del lenguaje de los niños y los hacen vulnerables a sufrir problemas de aprendizaje (Becket et al, 2006; Berástegui, 2005; Brodzinsky, Schechter y Henig, 1992; Dalen, 2002; Gunnar y Kertes, 2005; Juffer et al, 2005; Rutter, 2005; Van IJzendoorn y Juffer, 2006; van IJzendoorn, Juffer, y Klein Poelhuis, 2005).

Igualmente, la edad a la que fueron adoptados los menores también ha mostrado su influencia en su desarrollo cognitivo y su rendimiento escolar, ya que los niños adoptados en una edad más avanzada a menudo han estado expuestos por un periodo más largo, a factores negativos antes de la adopción (Dalen, 2007; Howe, 1997). Otros factores como los problemas de conducta pueden estar interviniendo también en este fenómeno. De hecho, en otros trabajos, las habilidades en el lenguaje y el nivel de comportamiento hiperactivo de los niños adoptados explicaron la mayor parte de la disparidad en el rendimiento escolar entre estos y los no adoptados (Dalen y Rygvold, 2006).

Para confirmar el peso de estas variables, numerosos trabajos han examinado, las diferencias entre niños adoptados y sus iguales en cuanto al funcionamiento cognitivo y el rendimiento escolar (Becket et al, 2006; Dalen, 2007; Lindblad, Dalen, Rasmussen, Vinnerljung y Hjern, 2009; Palacios, Sánchez-Sandoval y León, 2005; Vinnerljung, Lindblad, Hjern et al, 2010; Wilk, Loman, Van Ryzin et al, 2011). Así, por ejemplo, Beckett et al. (2006) encontraron que los niños con una edad de adopción de menos de 6 meses no presentaban un retraso del desarrollo cognitivo y sus puntuaciones de CI fueron similares en comparación con los niños adoptados a nivel nacional en el Reino Unido. Sin embargo, los niños adoptados después de 6 meses presentaron signos de un retraso del desarrollo cognitivo. Para Lindblad y su equipo, en Suecia, la adopción con más de cuatro meses se asoció con un menor logro educativo (Lindblad et al, 2003).

En el mismo sentido, un meta-análisis realizado por Van Ijzendoorn et al, (2005) basado en 62 estudios y 17767 niños adoptados, dirigido a examinar si el desarrollo cognitivo de los niños adoptados difería del de los niños que se quedaron al cuidado de instituciones o en la familia biológica y el de sus hermanos no adoptados o compañeros. Los niños adoptados puntuaron más alto en las pruebas de CI que sus hermanos no adoptados o compañeros que se quedaron en el mismo entorno y el rendimiento fue mejor. Sin embargo, aunque los niños adoptados no se diferenciaban de sus pares no adoptados actuales en el coeficiente in- 
telectual, su rendimiento escolar y las habilidades lingüísticas sí manifiestaban cierto retraso, derivando en problemas de aprendizaje. Resultados similares se obtuvieron en estudios españoles (Palacios y Sánchez, 1996), demostrando que la evolución de los adoptados había sido más positiva que la de sus iguales de origen (los menores que permanecieron en la institución o con sus familias de origen), aunque algo inferiores a los de sus iguales actuales.

Es importante señalar, en línea con lo anterior, que existen interesantes estudios longitudinales de adoptados que habían experimentado privación extrema temprana que han demostrado que los marcados efectos iniciales en cuanto a déficit cognitivo manifiestan una importante recuperación, aunque incompleta, con posterioridad (Beckett et al, 2006; Dalen, 2007; MacLean, 2003; Palacios et al, 2005). Tanto la pérdida inicial como el grado de recuperación varían en función de la duración y la gravedad de la privación experimentados (Beckett et al, 2006; O'Connor et al, 2000; Rutter et al, 1998). Los estudios también muestran como una intervención adecuada tanto en el seno de su nueva familia como en su entorno más inmediato puede permitir que superen estas dificultades (van IJzendoorn y Juffer, 2006; van IJzendoorn, Juffer y Poelhuis, 2005) y mejoren su rendimiento académico (Beckett et al, 2010).

En este trabajo -parte de una investigación más amplia sobre el proceso vivido por un grupo de familias adoptivas (Rosser, 2010)-, mostramos la evolución que siguieron un grupo de menores adoptados desde que se incorporaron a su nueva familia hasta el momento del estudio, tratando de averiguar si los hallazgos encontrados guardan relación con determinados aspectos de su experiencia previa a la adopción.

\section{Objetivos}

En concreto, los objetivos de esta investigación han sido:

1) Evaluar las dificultades que presentaban los menores a la llegada a su nueva familia, especialmente en el ámbito del desarrollo cognitivo-lingüístico y escolar, y su evolución hasta el momento del estudio.

2) Analizar posibles factores influyentes en la aparición y persistencia de estas dificultades.

3) Proponer actuaciones para favorecer la adaptación de los menores a la escuela. 


\section{Método}

\section{Participantes}

El estudio se ha realizado con todos los menores españoles que fueron adoptados en Alicante (España) entre 1996 y 2001, un total de 133 menores entre 0 y 12 años, con una media de cuatro años y medio de convivencia con sus familias adoptivas. De la totalidad de la muestra colaboraron en la realización de los cuestionarios las familias correspondientes a 89 casos. Posteriormente, para evaluar la adaptación escolar, excluimos los casos que no cumplían el criterio de estar en edad escolar (mayores de 2 años al ser adoptados), quedando la muestra final reducida a 57 casos.

\section{Instrumentos}

Se utilizó una ficha diseñada ad hoc para obtener la infomación recogida en los expedientes de los menores en la que se reunieron los datos sobre el menor (sexo, edad, y características especiales), sobre la problemática familiar que motivó la separación del menor de su familia biológica, sobre las medidas adoptadas (cuando accedieron al sistema de protección, qué medidas se adoptaron, $\mathrm{n}^{\mathrm{o}}$ de centros, tiempo de permanencia, acogimientos familiares previos, etc.) y también datos de la familia adoptiva (edad, estudios, profesión, otros hijos, etc.). Asimismo, con el objetivo de recoger información sobre los problemas que presentaban los niños y niñas a su llegada y los que persistían en el momento del estudio se realizaron entrevistas con los padres y madres de estos menores.

Finalmente, se utilizó la Escala para la detección de problemas en la adaptación de los menores adoptados de Fernández y Fuentes (2000), en la cual los padres debían indicar la presencia de dificultades al inicio del acogimiento y su posible persistencia en el momento del estudio. La escala consta de 40 ítems, divididos en 4 áreas: aspectos relacionados con la salud y el desarrollo físico, sobre el desarrollo cognitivo y lingüístico, sobre el desarrollo afectivo y emocional y sobre el establecimiento de relaciones sociales y asunción de normas.

\section{Procedimiento}

En una primera fase acudimos a la entidad pública responsable del programa de adopción para examinar los expedientes y recabar información sobre los datos y problemática de los menores. En la segunda fase, se relizaron las entrevistas con las famlias y poste- 
riormente, éstas cumplimentaron la Escala para la detección de problemas en la adaptación de los menores adoptados de Fernández y Fuentes (2000). Las familias debían señalar si habían detectado determinados problemas en sus hijos al inicio de la convivencia y si estos persistían en el momento del estudio.

\section{Análisis Estadístico}

Los estadísticos descriptivos utilizados en esta fase de la investigación han sido las frecuencias y porcentajes obtenidos en cada una de las variables estudiadas, así como la media y desviación típica, cuando se trataba de variables cuantitativas. Para comprobar si existían diferencias antes y después de la escolarización utilizamos diversas pruebas no paramétricas (Wilconxon y Friedman) para muestras relacionadas. Finalmente, se fectuaron correlaciones para determinar si existía alguna relación entre las dificultades detectadas y su persistencia y determinadas características de los menores y su experiencia previa se utilizó el coeficiente de Spearman. Para el análisis estadístico se ha utilizado el SPSS 15.0.

\section{Resultados}

En el estudio de partida a partir del cual se analizan estos datos (Rosser, 2010) se evidenciaba la complejidad de los casos que llagaban a adopción. La mayoría de ellos (el 95\% de los casos) derivaban de situaciones de desprotección, de maltrato y/o abandono, de problemáticas de desestructuración familiar, violencia, toxicomanías, etc. y habían estado sometidos a largos periodos de institucionalización (el 36. 5\% de los casos permanecieron más de 1 año en centros).

En esta parte de la investigación, centrada en las dificultades que presentaban los menores en el momento de su acogimiento preadoptivo y su evolución posterior, comprobamos que los menores adoptados presentaban a su llegada a la nueva familia dificultades en diferentes ámbitos (Tabla 1). 
Tabla 1. Resultados de la Escala de detección de problemas infantiles (Fernández y Fuentes, 2000).

\begin{tabular}{|c|c|c|c|c|}
\hline \multirow[t]{2}{*}{ Problemas infantiles } & \multicolumn{2}{|c|}{ Aparicion } & \multicolumn{2}{|c|}{ Persistencia } \\
\hline & $\mathrm{N}$ & $\%$ & $\mathrm{~N}$ & $\%$ \\
\hline \multicolumn{5}{|l|}{ Estado de salud y desarrollo } \\
\hline 1. Desnutrición, peso y talla bajos, retraso dentición, etc. & 30 & 36.1 & 3 & 3.6 \\
\hline 2. Problemas dentales, infecciones, alergias, etc. & 30 & 35.7 & 14 & 16.7 \\
\hline 3. Pesadillas, dormir poco. & 23 & 28 & 8 & 9.8 \\
\hline 4. Falta de control de esfínteres. & 27 & 32.9 & 7 & 8.5 \\
\hline 5. Vómitos, mareos, malestar sin causa orgánica. & 6 & 7.1 & 2 & 2.4 \\
\hline \multicolumn{5}{|l|}{ Desarrollo cognitivo-linguistico y ajuste escolar } \\
\hline 6. Pronunciación deficiente, dislalias. & 24 & 29.3 & 6 & 7.3 \\
\hline 7. Falta de léxico, desconocimiento de palabras. & 33 & 40.2 & 10 & 12 \\
\hline 8. Retraso escolar de uno o más cursos. & 25 & 30.9 & 16 & 20 \\
\hline 9. Ansiedad ante las tareas escolares, pereza, nerviosismo. & 31 & 38.8 & 24 & 29.6 \\
\hline \multicolumn{5}{|l|}{ Desarrollo afectivo y emocional } \\
\hline 10. Recuerdos frecuentes de sus otros hermanos. & 8 & 9.8 & 0 & 0 \\
\hline 11. No expresa afecto a sus padres adoptivos. & 16 & 20 & 6 & 7.5 \\
\hline 12. Miedo a perder a esta familia. & 40 & 51.3 & 12 & 15.6 \\
\hline 13. Autoagresiones físicas, tirarse del pelo, golpearse. & 7 & 8.8 & 2 & 2.5 \\
\hline 14. Autoagresiones verbales, insultarse. & 5 & 6 & 3 & 3.6 \\
\hline 15. Acapara objetos, juguetes, los esconde. & 17 & 20.2 & 10 & 11.9 \\
\hline 16. Acapara comida, la guarda para después. & 16 & 19 & 8 & 9.5 \\
\hline 17. Niega su pasado. & 12 & 15.4 & 6 & 7.6 \\
\hline 18. Miedo a que sepan que es adoptado. & 4 & 4.9 & 3 & 3.7 \\
\hline 19. Confunde su historia, fantasea con su pasado. & 17 & 21.5 & 4 & 5.2 \\
\hline 20. Recuerdos del pasado que le crean inquietud. & 24 & 31.2 & 8 & 10.3 \\
\hline 21. Excesiva curiosidad sexual hacia los padres. & 7 & 8.5 & 2 & 2.5 \\
\hline 22. Conductas sexuales con otros niños/as. & 8 & 9.8 & 1 & 1.2 \\
\hline 23. Pudor y ocultamiento del propio cuerpo. & 6 & 7.2 & 5 & 6.1 \\
\hline \multicolumn{5}{|l|}{ Relaciones sociales y normas } \\
\hline 24. Inhibición social. & 5 & 6 & 1 & 1.2 \\
\hline 25. Agresividad verbal hacia adultos. & 7 & 8.3 & 4 & 4.8 \\
\hline 26. Agresividad física hacia adultos. & 3 & 3.6 & 2 & 2.4 \\
\hline 27. Agresividad verbal hacia iguales. & 8 & 9.5 & 7 & 8.3 \\
\hline 28. Agresividad física hacia iguales. & 8 & 9.5 & 7 & 8.3 \\
\hline 29. Amistades con niños más pequeños. & 36 & 43.4 & 24 & 31.2 \\
\hline 30. Mentiras. & 27 & 33.3 & 19 & 24.1 \\
\hline 31. Pequeños robos. & 19 & 23.5 & 6 & 7.4 \\
\hline 32. No cumple algunas tareas domésticas asignadas. & 25 & 31.3 & 21 & 26.3 \\
\hline 33. No cumple las normas en la comida. & 18 & 22 & 12 & 14.8 \\
\hline 34. No cumple las normas de higiene. & 21 & 26.6 & 13 & 16 \\
\hline 35. Rechazo de todo tipo de normas, negativismo. & 13 & 16 & 7 & 8.6 \\
\hline 36. Celos, rivalidad con los hermanos. & 20 & 24.7 & 19 & 23.8 \\
\hline 37. Peleas, agresividad con hermanos. & 11 & 13.6 & 8 & 9.9 \\
\hline 38. Rabietas. & 35 & 43.8 & 24 & 30.4 \\
\hline 39. Llamadas constantes de atención. & 38 & 46.3 & 31 & 38.8 \\
\hline 40. Otros. & 3 & 8.6 & 1 & 3.2 \\
\hline
\end{tabular}


Los resultados indican que, más de la tercera parte presentaban problemas físicos y de salud, posiblemente derivados de la falta de atención en sus primeros años de vida como problemas de peso y talla $(\mathrm{n}=30 ; 36 \%)$, problemas dentales, inmunodeficiencia y propensión a las infecciones $(n=30 ; 35,7 \%)$, etc. así como problemas de sueño $(n=23 ; 28 \%)$ o en el control de esfínteres $(n=27 ; 33 \%)$.

En un porcentaje similar, las familias detectaron problemas relacionados con su desarrollo cognitivo y lingüístico y su ajuste escolar. Los menores, a su llegada a la nueva familia, presentaban en un porcentaje importante, retrasos en el desarrollo del lenguaje, expresados por una pobreza de vocabulario $(n=33 ; 40 \%)$ o trastornos en la articulación $(n=24$; $29 \%$ ). Igualmente manifestaban retraso en los conocimientos adquiridos, probablemente fruto de la falta de estimulación, la asistencia irregular a la escuela, etc. con un retraso de uno o más cursos escolares $(\mathrm{n}=25 ; 31 \%)$, así como dificultad para organizarse las tareas y para prestar atención en clase, lo que les generaba, consiguientemente, ansiedad ante la realización de las tareas escolares o la propia asistencia al centro, pereza o nerviosismo $(n=31$; $39 \%)$.

Un área también afectada del desarrollo psicosocial era la que tenía que ver con su desarrollo afectivo y emocional, manifestándose en forma de inseguridad afectiva o miedo a perder a la familia $(\mathrm{n}=40 ; 51 \%)$, acaparar objetos $(\mathrm{n}=17 ; 20 \%)$, fantasear sobre su pasado $(n=17 ; 21 \%)$, etc.), o dificultades en el establecimiento de nuevos vínculos $(n=16 ; 20 \%)$. Por otra parte, destacaba el número de problemas relacionales y de conductas inadecuadas, como los pequeños robos $(n=19 ; 23,5 \%)$, las mentiras $(n=29 ; 33 \%)$, las rabietas $(n=35 ; 44 \%)$, llamadas de atención $(n=38 ; 46 \%)$ o la agresividad, $(n=11 ; 13 \%)$. Un dato relevante de la investigación ha sido la constatación a través de la prueba de Wilconson de que, en el momento del estudio, el número medio de problemas detectados al inicio de la convivencia $(\mathrm{M}=8.01, \mathrm{dt}=7.895)$ ha disminuido significativamente al compararlos con el momento del estudio $(\mathrm{M}=4.08, \mathrm{dt}=5.23, \mathrm{Z}=-6.228 ; \mathrm{p}=.000)$.

El contraste realizado a través de la prueba de Friedman indica diferencias entre el inicio de la convivencia y en el momento del estudio que son estadísticamente significativas en todas las áreas. Así, podemos comprobar cómo las medias en la manifestación de dificultades en las distintas áreas han disminuido significativamente durante el tiempo de permanencia de los menores con su familia adoptiva (Figura 1). 


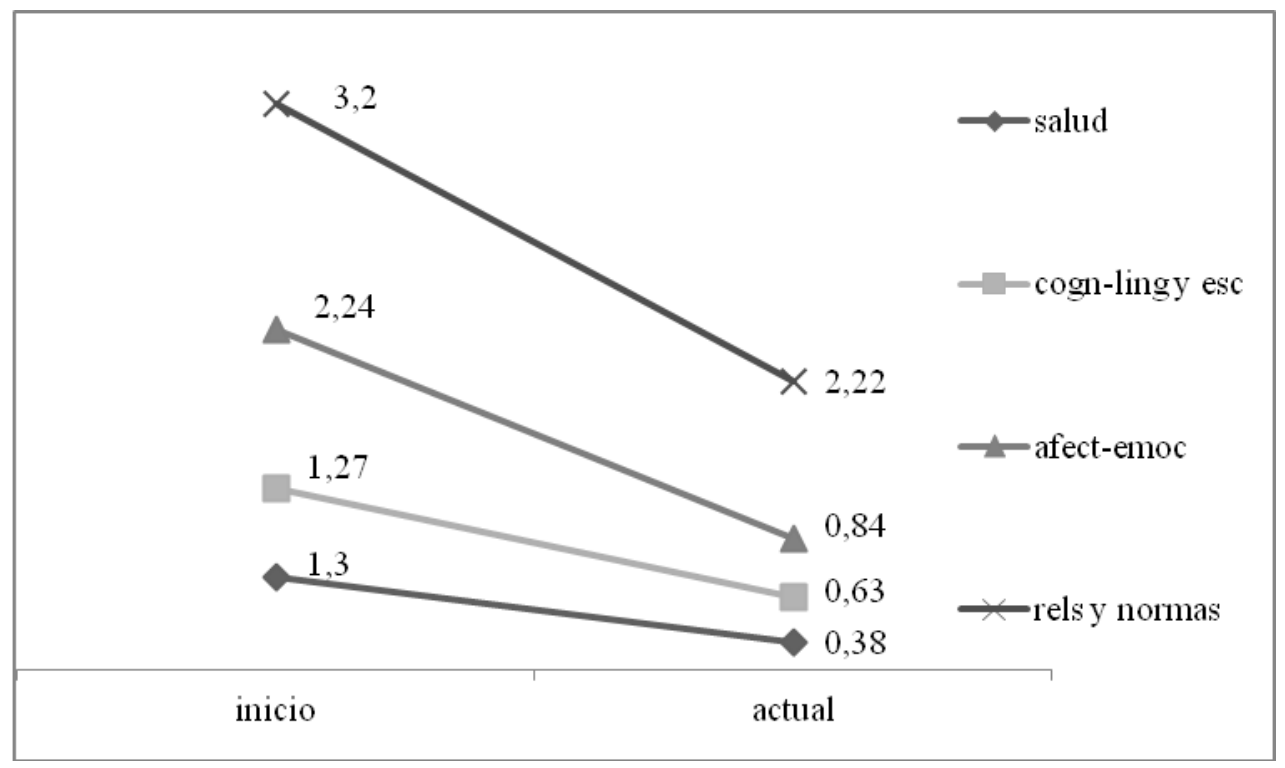

Figura 1. Evolución de las dificultades desde la incorporación a la nueva familia hasta el momento del estudio.

Estas diferencias (tabla 2) son estadísticamente significativas en el desarrollo físico y la salud protección $\left(\chi_{(1)}^{2}=43,085, \mathrm{p}=.000\right)$, en los aspectos relacionados con el desarrollo cognitivo lingüístico y escolar $\left(\chi_{(1)}^{2}=33,000, p=.000\right)$, en lo referente a su seguridad afectiva y sus emociones $\left(\chi_{(1)}^{2}=32,362, p=.000\right)$, y en el comportamiento relacionado con las relaciones sociales y el cumplimiento de normas $\left(\chi_{(1)}^{2}=22,261, \mathrm{p}=.000\right)$.

Tabla 2: Problemas infantiles detectados al inicio de la convivencia y en el momento del estudio en las distintas áreas.

\begin{tabular}{lccccc}
\hline Tipo de problema & Media & $\begin{array}{c}\text { Desviación } \\
\text { típica }\end{array}$ & $\mathrm{X}^{2}$ & gl & Sig. asint. \\
\hline Aparición problemas salud & 1,30 & $(1,369)$ & 43,08 & 1 &, 000 \\
Persistencia problemas salud &, 38 & $(, 666)$ & & & \\
Aparición problemas cognitivo-lingüístico y & 1,27 & $(1,404)$ & 33,00 & 1 &, 000 \\
Persistencia problemas cognitivo-lingüístico y &, 63 & $(, 970)$ & & & \\
Aparición problemas afectivo y emocional & 2,24 & $(2,697)$ & 32,36 & 1 &, 000 \\
Persistencia problemas afectivo y emocional &, 84 & $(1,397)$ & & & \\
Aparición problemas relaciones y normas & 3,20 & $(3,501)$ & 22,26 & 1 &, 000 \\
Persistencia problemas relaciones y normas & 2,22 & $(2,969)$ & & & \\
\hline
\end{tabular}

a Prueba de Friedman

Cuando relacionamos estos resultados con los antecedentes del menor se comprueba que determinados hitos de la historia previa de los menores están influyendo en estos resultados (Tabla 3). Por ejemplo, la edad a la que ingresaron los menores en centros de protec- 
ción $\left(\chi_{(2)}^{2}=23,221, p=.000\right)$, el tiempo que estuvieron institucionalizados $\left(\chi_{(4)}^{2}=13,005\right.$, $\mathrm{p}=.011)$, y la edad al inicio del acogimiento preadoptivo $\left(\chi_{(2)}^{2}=22,552, \mathrm{p}=.000\right)$, han tenido un peso significativo en la aparición de estos problemas.

Igualmente, las experiencias previas de los menores están influyendo en la persistencia de este tipo de problemas (Tabla 3). En este caso vemos cómo cuanto más mayores llegaron los menores a los centros de protección $\left(\chi_{(2)}^{2}=9,552, p=.009\right)$, más tiempo permanecieron institucionalizados $\left(\chi_{(4)}^{2}=14,352, \mathrm{p}=.006\right)$, y más mayores eran al inicio del acogimiento preadoptivo $\left(\chi_{(2)}^{2}=12,995, \mathrm{p}=.002\right)$, se producía una mayor resistencia a la resolución de estas dificultades.

Tabla 3: Relación entre los antecedentes del menor y la aparición y persistencia de problemas durante el periodo de adaptación.

\begin{tabular}{ccccccc}
\hline & \multicolumn{3}{c}{ Aparición } & \multicolumn{3}{c}{ Persistencia } \\
\hline & $\mathrm{X}^{2}$ & $\mathrm{gl}$ & Sig. asint & $\mathrm{X}^{2}$ & $\mathrm{gl}$ & Sig. asint \\
Edad inicio internamiento & 23,221 & 2 & .000 & 9,522 & 2 & .009 \\
Tiempo internamiento & 13,005 & 4 &, 011 & & 4 &, 006 \\
Edad inicio acogimiento & 22,552 & 2 & .000 & 12,995 & 2 & .001 \\
\hline
\end{tabular}

a. Kruskal Wallis Test;

b. ** La correlación es significativa al nivel 0,$01 ; *$ La correlación es significante al nivel 0,05

Por otra parte, centrándonos en el ámbito que nos ocupa, el desarrollo cognitivolingüístico de estos menores y sus ajuste escolar vemos que, cuando analizamos los resultados en esta área con los 57 menores que habían iniciado la escolarización en el momento del estudio se repite el fenómeno, de forma que los problemas que en un principio mostraban al inicio de la convivencia $(\mathrm{M}=1,77, \mathrm{dt}=1.41)$, se han reducido significativamente tras la convivencia $(\mathrm{M}=0.93, \mathrm{dt}=1.08), \mathrm{Z}=-4.362, \mathrm{p}=.000$.

Igualmente se comprueba que existe una relación entre los problemas cognitivolingüísticos y de ajuste escolar que presentan estos menores y aquellos de carácter afectivoemocional o aquellos que tienen que ver con el comportamiento de los menores en las relaciones con los demás y en la asunción de normas. De hecho, es observable cómo la aparición de problemas cognitivo-lingüísticos y escolares correlaciona con la aparición de problemas afectivo-emocionales $\left(\mathrm{r}=, 668^{* * *}\right)$ y los que tienen que ver con las relaciones sociales y las normas $\left(\mathrm{r}=, 645^{* * *}\right)$. Igualmente la persistencia de los problemas cognitivo- 
lingüísticos y escolares correlaciona tanto con la persistencia de problemas afectivoemocionales $(\mathrm{r}=, 439 * *)$ cómo con los que tienen que ver con las relaciones sociales y las normas $(\mathrm{r}=, 488 * * *)$.

\section{Discusión}

El presente estudio tiene el valor de recoger la información referente a la totalidad de los casos de menores que fueron adoptados en el ámbito provincial de Alicante a través de una adopción nacional entre los años 1996 y 2001. Sin embargo, al tener un ámbito provincial, los resultados deben ser tratados con cautela a la hora de generalizarlos a otros contextos. En cualquier caso, los datos permiten hacer observaciones de interés sobre la adaptación al ámbito escolar de los menores tras su adopción.

Si los adoptados han de realizar un gran esfuerzo para adaptarse a un nuevo entorno familiar, a nuevas rutinas, casi a la par, han de adaptarse a un nuevo entorno escolar. En la escuela se ponen de manifiesto las posibles carencias que arrastran como consecuencia de haber crecido en entornos desfavorecidos. Estas dificultades fueron descritas por Gindis (2000, 2006) como el Déficit cognitivo acumulado (Cumulative Cognitive Deficit) que consiste en una tendencia a la baja en la medida de la inteligencia y el rendimiento escolar de los niños socialmente desfavorecidos en relación con la edad. Para este autor, la comprensión actual del CCD es que los niños que están privados de experiencias cognoscitivas enriquecedoras durante sus primeros años tienen menos posibilidades de beneficiarse de una situación nueva y enriquecedora del entorno a causa de un desfase entre la madurez cognitiva y los requisitos estructurales para un aprendizaje más avanzado propios de estas nuevas situaciones. Además, el CCD se asocia generalmente, según Gindis (2000) con ciertos problemas emocionales y/ conductuales al provocar una espiral en la que el fracaso en los esfuerzos cognoscitivos genera una baja autoestima, falta de interés y frustración constante. La falta de motivación intrínseca en las actividades cognoscitivas crece con la edad y se convierte en una de las principales características de la CCD.

Los datos de la investigación nos indican en primer lugar la presencia de dificultades en los menores a su llegada a la nueva familia, dificultades que se manifiestan en diferentes ámbitos cómo la salud, el desarrollo cognitivo y del lenguaje, el desarrollo afectivo y emocional, las relaciones sociales o el comportamiento y cumplimiento de normas, y que ponen 
en evidencia la vulnerabilidad de los menores adoptados en su proceso de adaptación (Beckett et al, 2006; Berástegui, 2005; Brodzinsky, 1990; Dalen, 2007; Juffer et al, 2005; O’Connor et al, 2000; Rutter, 2005; Zeanah et al, 2003).

Ahondando en las posibles razones de estas dificultades, los datos muestran, en línea con otros autores (Juffer y van Ijzendoorn, 2005; O'Connor et al, 2000; Rutter, 2005; Verhulst et al, 1990, 1992; van IJzendoorn y Juffer, 2006; van IJzendoorn et al, 2005), que las experiencias previas de los menores han dejado su influencia en su desarrollo psicosocial, tanto más según la edad a la que ingresaron en el sistema de protección, el tiempo que permanecieron institucionalizados, o la edad a la que iniciaron la convivencia con su familia adoptiva. De hecho, aquellos menores que ingresan más mayores, habiéndose visto expuestos por más tiempo a situaciones adversas para su desarrollo, y que, en consecuencia, llegaron más tarde a sus nuevas familias, arrastraban más dificultades que aquellos que fueron extraídos más tempranamente de estos entornos adversos y por lo tanto encontraron también más tempranamente una estabilidad en su nueva familia. Además, los resultados del estudio indican que estas variables están teniendo una influencia clara tanto en la aparición como en la persistencia de estos problemas.

En concreto, cuando nos centramos en las dificultades de índole cognitivo-lingüística y de ajuste escolar, encontramos que la tercera parte de los menores presentaban dificultades en esta área. Se trata de niños y niñas que presentaban un lenguaje pobre, con falta de léxico. A menudo mostraban dificultades para seguir el ritmo escolar, cursaban con uno o más años de retraso y/o manifestaban rechazo hacia las tareas escolares. Además estos problemas se mantenían en un número significativo en el momento del estudio.

Otro hallazgo importante ha sido constatar que estos problemas no suelen darse solos sino que van acompañados de problemas de otro tipo como los afectivo-emocionales y los relacionados con la conducta del menor en sus relaciones con los demás y en el cumplimiento de las normas. En nuestro estudio se comprueba que existe una interrelación entre los diferentes tipos de problemas, especialmente entre los comportamentales, emocionales y cognitivo-lingüísticos y escolares, de forma que la presencia de unos provoca o acentúa la presencia de otros. Los resultados indican, además, que la interferencia entre los diferentes tipos de problemas persiste en el momento del estudio. 
En consonancia con otras investigaciones (Dalen y Rygvold, 2006), estas interferencias podrían estar ocasionando dificultades en su aprendizaje y ajuste escolar que a menudo persisten e incluso pueden empeorar con el tiempo. Lógicamente, un menor que manifiesta ansiedad ante la separación de su familia, que fantasea sobre su pasado, que trata de llamar constantemente la atención con su comportamiento, que presenta rabietas, negativismo, o mentiras, que no cumple las tareas asignadas o realiza pequeños hurtos, esconde objetos del aula, etc., como hemos visto que ocurre en porcentajes significativos con los menores del estudio, va a ver como queda comprometido su proceso de adaptación en la escuela. De la misma forma, los problemas de lenguaje, la ansiedad ante las tareas escolares, y los problemas de rendimiento escolar que se deriven de ello, pueden inducir a conductas inadecuadas en el menor, fruto de su frustración y falta de motivación, que también afectarán a su integración con compañeros y profesores.

Estas dificultades que afectan al ajuste escolar y que relacionamos con el Déficit cognitivo acumulado (CCD) descrito por Giddins, van a seguir afectando, si no se actúa para compensarlas, tanto a los logros académicos del menor como a aspectos emocionales y comportamentales, manifestándose cada vez de forma más evidente conforme los menores avanzan en los ciclos formativos. En este sentido, los resultados de este trabajo reflejan, en sintonía con diversas investigaciones (Beckett et al, 2006; Dalen, 2007; MacLean, 2003) que los menores tienden a ir resolviendo estos problemas tras la adopción, aunque esta recuperación no es completa. Tal y cómo como han señalado otros autores (Fernández el al, 2000; O’Connor et al, 2000; Palacios et al, 1996; Rutter, 2005), en este trabajo se constata cómo, aún cuando en el grupo estudiado las dificultades se reducen, los problemas no llegan a resolverse del todo. Se trata de problemas resistentes y que requerirían de una mayor atención.

La evidencia de estas dificultades y de la complejidad de los factores influyentes en las mismas nos lleva a hacer hincapié en la importancia de proporcionar a estos menores un entorno protector y potenciador de sus competencias y habilidades para facilitar su recuperación e integración escolar, y pone de manifiesto la necesidad de coordinar las actuaciones entre la familia y la escuela para prevenir y detectar precozmente estas dificultades, facilitando conjuntamente los medios para su adecuada resolución. En este sentido, es crucial sensibilizar a maestros y educadores ante estos nuevos retos, cuidar la incorporación a la escuela de los menores tras la adopción, que familia y escuela se coordinen en el abordaje de 
las posibles dificultades, que se respete el ritmo de los menores en su adaptación a este nuevo entorno y que se cuente con servicios especializados de asesoramiento y apoyo.

En todas estas tareas tienen un papel destacado para prevenir y/o abordar precozmente las dificultades de adaptación tanto académica como social de los menores adoptados los Servicios Post adoptivos, en coordinación con el equipo educativo y con las familias (Rosser, Bueno y Suriá, 2011). Destacan en esta línea experiencias como las desarrolladas en nuestro país (España), desde diversas administraciones y entidades como Agintzari (2006) en el País Vasco; Labajo y Bueno (2008) en Castilla y León; Parrondo (2007) en Madrid, orientadas a apoyar el proceso de adaptación de los menores adoptados a la escuela y al desarrollo de servicios especializados de asesoramiento y apoyo.

\section{Referencias}

Agintzari (2006). Guía de postadopción para profesionales de la educación y agentes sociales. Vitoria Gasteiz: Servicio de Publicaciones del Gobierno vasco.

Beckett, C., Maughan, M. Rutter, M., Castle, J., Colvert, E., Groothues, C., Kreppner, J., Stevens, S, O'Connor, T. y Sonuga-Barka, E.J.S. (2006). Do the effects of early severe deprivation on cognition persist into early adolescence? Findings from the English and Romanian adoptees study. Child Development, 77, 3, 696-711.

Berástegui, A. (2005). La adaptación familiar en adopción internacional. Una muestra de adoptados mayores de tres años en la Comunidad de Madrid. Madrid. Consejo Económico y Social.

Berástegui, A. (2006). La adaptación escolar de los adoptados internacionales: una cuestión emergente. En M.I. Álvarez y A. Berástegui (cords). Educación y familia: la educación familiar en un mundo en cambio. Madrid: Universidad Pontificia de Comillas.

Brodzinsky, D.M (1987). Adjustment to adoption: A psychosocial perspective. Clinical Psychology Review, 7 (1), 25-47.

Brodzinsky D.M., Schechter, D.E., Braff A.M. y Singer, L.M. (1984). Psychological and academic adjustment in adopted children. Journal of Consulting and Clinical Psychology, $54,582-590$. 
Colombo, M., de la Parra, A. y López, I. (1992). Intellectual and physical outcome of children undernourished in early life is influenced by later environment conditions. Developmental Medicine and Child Neurology, 34, 611-622.

Croft, C., Beckett, C., Rutter, M., Castle, J., Colvert, E., Groothues, C., et al. (2007). Early adolescent outcomes of institutionally-deprived and non-deprived adoptees. II: Language as a protective factor and a vulnerable outcome. Journal of Child Psychology and Psychiatry, 48, 31-44.

Dalen, M. (2001). School performances among internationally adopted children in Norway. Adoption Quarterly, 5(2), 39-58.

Dalen, M. (2005). International adoptions in Scandinavia: Research focus and main results. En D. Brodzinsky y J. Palacios (Eds.), Psychological Issues in Adoption. Research and Practice (pp. 187-211). USA: Praeger.

Dalen, M. y Rygvold, A.L. (2006). Educational achievement in adopted Children from China. Adoption Quarterly, 9(4), 45-58.

Dalen, M., Hjern, A., Lindblad, F., Odenstad, A., Rasussen, F. y Vinnerljung, B. (2008). Educational attainment and cognitive competence in adopted men. A study of international and national adoptees, siblings and a general Swedish population. Children and Youth Services Review, 30, 1211-1219.

Dalen, M. (2007). Educational achievement among international adoptees. Anuario de Psicología, 38(2), 199-208.

Fernández, M. y Fuentes, M. J. (2000). Variables infantiles de riesgo en el proceso de adaptación de niños/as de adopciones especiales. Infancia y Aprendizaje, 95, 341-359.

Freixat, M. (2009). Adopción y escuela. Conferencia presentada en Getafe organizada por la Asociación Atlas. Disponible en http://www.asatlas.org/descargas/cat_view/52jornadas

Gindis, B. (1998). Navigating uncharted Waters: school psychologist working with internationally adopted postinstitutionalized children (Part I). Comuniqué, 27, 6-9.

Gindis, B. (2000). Detecting and Remediating the Cumulative Cognitive Deficit in School Age Internationally Adopted Post-institutionalized Children. The Parent Network for the Post-Institutionalized Child, 27 (1), 1-6. Disponible en: http://www.bgcenter.com/CCDPost.htm 
Gindis, B. (2006). Cumulative cognitive deficit in international adoptees: its origin, indicators, and means of remediation. The Family Focus, FRUA (Families for Russian and Ukrainian Adoptions) newsletter, 12 (1), 1-2 y 12 (2), 6-7. Disponible en: http://www.bgcenter.com/BGPublications/CCD_in_international_adoptees.htm

Howe, D. (1997). Parent - reported problems in 211 adopted children: Some risk and protective factors. Journal of Child Psychology and Psychiatry and Allied Disciplines. 38(4), 401-411.

Kovas, Y., Haworth, C. M. A., Dale, P. S. y Plomin, R. (2007). The genetic and environmental origins of learning abilities and disabilities in the early school years. Monographs of the Society for research in Child Development, 72, 1-144.

Labajo, G. y Bueno, N. (2008). Guía didáctica en materia de adopción para educación infantil y primaria. Junta de Castilla y León. Disponible en: http://www.educa.jcyl.es/educacyl/cm/educacyl/tkContent?pgseed=1240389165963\&i $\underline{\mathrm{dContent}}=80422 \&$ locale $=$ es_ES\&textOnly $=$ false

Lindblad, F., Dalen, M., Rasmussen, F., Vinnerljung, B. y Hjern, A. (2009). School performance of international adoptees better than expected from cognitive test results. European Child and Adolescent Psychiatry, 18, 301-308.

MacLean, K. (2003). The impact of institutionalization on child development. Development and Psychopathology, 15, 853-884.

Martínez-Monteagudo, M.C., Inglés, C., Trianes, M.V. y García-Fernández, J.M. (2011). Perfiles de ansiedad escolar: Diferencias en clma social y violencia entre iguales. Electronic Journal of research in Educational psychology, 9(3), 1023-1042.

O’Connor, T. G., Rutter, M., Beckett, C., Keaveney, L., Kreppner, J. y the ERA study team. (2000). The effects of global severe privation on cognitive competence: Extension and longitudinal follow-up. Child Development, 71, 376-390.

Palacios, J. y Sánchez, Y. (1996). Niños adoptados y no adoptados: un estudio comparativo. Anuario de Psicología, l, 63-85.

Palacios, J., Sánchez-Sandoval, Y. y León, E. (2005). Adopción Internacional en España: un nuevo país, una nueva vida. Madrid: Ministerio de Trabajo y Asuntos Sociales. 
Parrondo, L. (coord) (2007). Adoptar, Integrar y Educar. Una Guía de orientación para educadores y familias. Madrid: Instituto Madrileño del Menor y la Familia, Comunidad de Madrid. (2a edición).

Suriá, R. (2011). Análisis comparativo sobre las actitudes de los estudiantes hacia sus compañeros con discapacidad. Electronic Journal of research in Educational psychology, 9(1), 197-216

Rosser, A. (2010). Evolución del proceso de adopción y satisfacción percibida por las familias adoptivas. Valencia: Corts Valencianes.

Rosser, A., Bueno, A. y Suriá, R. (2011). El apoyo postadoptivo como instrumento de prevención de los problemas de integración socioeducativa de los menores adoptados. En Roman, Carbonero y Valdivieso, (comp.). Educación, aprendizaje y desarrollo en una sociedad multicultural. Madrid: Ediciones de la Asociación de Psicología y Educación, 9823-9833

Rutter, M. (1985) Family and school influences on cognitive development. Journal of Child Psychology and Psychiatry, 26(5), 683-704.

Rutter, M. (2005). Adverse preadoption experiences and psychological outcomes. En D. Brodzinsky y J. Palacios (Eds.), Psychological Issues in Adoption. Research and Practice, 47-67). USA: Praeger.

van IJzendoorn M.H. y Juffer F. (2006). The Emanuel Miller Memorial Lecture 2006: Adoption as intervention. Meta-analytic evidence for massive catch-up and plasticity in physical, socio-emotional, and cognitive development. Journal of Child Psychology and Psychiatry, 47, 1228-1245.

van Ijzendoorn, M.H., Juffer, F. y Klein Poelhuis, C.W (2005). Adoption and cognitive development: A metaanalytic comparison of adopted and nonadopted children's IQ and school performances. Psychological Bulletin, 131, 301-316.

Verhulst, F.C., Althaus, M. y Verluis-den Bierman, H. (1990). Problem behaviour in international adoptees. Journal of American Academy of Child and Adolescent Psychiatry, 29, $94-103$.

Verhulst, F.C., Althaus, M. y Verluis-den Bierman, H. (1992). Damaging background: Later adjustment of international adoptees. Journal of American Academy of Child and Adolescent Psychiatry, 31, 518-524. 
Zeanah, C.H., Nelson, C.A., Fox, N.A., Smyke, A.T, Marshall, P. Parker, S.W. y Koga, S. (2003). Designing research to study the effects of institutionalization on brain and behavioural development: The Bucharest Early Intervention Project. Development and Psychopathology, 15, 885-907. 